\title{
Hydrogen-Free Liquid-Helium Recovery Plants: The Solution for Low-Temperature Flow Impedance Blocking
}

\author{
M. Gabal, ${ }^{1,2}$ A. Arauzo, ${ }^{3}$ A. Camón,,${ }^{1,4}$ M. Castrillo, ${ }^{3}$ E. Guerrero, ${ }^{3}$ M. P. Lozano, ${ }^{1,2}$ M. P. Pina, ${ }^{2}$ J. Sesé, ${ }^{1,2}$ \\ S. Spagna, ${ }^{5}$ J. Diederichs ${ }^{5}$ G. Rayner, ${ }^{5}$ J. Sloan, ${ }^{5}$ F. Galli, ${ }^{6}$ W. van der Geest ${ }^{6}$ C. Haberstroh, ${ }^{7}$ \\ N. Dittmar, ${ }^{7}$ A. Oca, ${ }^{8}$ F. Grau, ${ }^{8}$ A. Fernandes, ${ }^{8}$ and C. Rillo ${ }^{1,4}, *$ \\ ${ }^{1}$ Departamento de Física de la Materia Condensada, Universidad de Zaragoza, Zaragoza 50009, Spain \\ ${ }^{2}$ Instituto de Nanociencia de Aragón (INA), Universidad de Zaragoza, Zaragoza 50018, Spain \\ ${ }^{3}$ Servicio General de Apoyo a la Investigación-SAI, Universidad de Zaragoza, Zaragoza 50009, Spain \\ ${ }^{4}$ Instituto de Ciencia de Materiales de Aragón (ICMA), Universidad de Zaragoza-CSIC, \\ Zaragoza 50009, Spain \\ ${ }^{5}$ Quantum Design Inc., San Diego, California 92121, USA \\ ${ }^{6}$ Leiden Institute of Physics, Leiden 2333 CA, The Netherlands \\ ${ }^{7}$ Technische Universität Dresden, 01062 Dresden, Germany \\ ${ }^{8}$ C.P.G.E. Air Liquide España, Madrid 28021, Spain \\ (Received 14 March 2016; revised manuscript received 2 June 2016; published 26 August 2016)
}

\begin{abstract}
The blocking of fine-capillary tubes used as flow impedances in ${ }^{4} \mathrm{He}$ evaporation cryostats to achieve temperatures below $4.2 \mathrm{~K}$ is generally attributed to nitrogen or air impurities entering these tubes from the main bath. The failure of even the most rigorous low-temperature laboratory best practices aimed at eliminating the problem by maintaining the cleanliness of the helium bath and preventing impurities from entering the capillary tubes suggests that a different cause is responsible for the inexplicable reduction of impedance flow. Many low-temperature research laboratories around the world have suffered this nuisance at a considerable financial cost due to the fact that the affected systems have to be warmed to room temperature in order to recover their normal low-temperature operation performance. Here, we propose an underlying physical mechanism responsible for the blockages based upon the freezing of molecular $\mathrm{H}_{2}$ traces present in the liquid-helium bath. Solid $\mathrm{H}_{2}$ accumulates at the impedance low-pressure side, and, after some time, it produces a total impedance blockage. The presence of $\mathrm{H}_{2}$ traces is unavoidable due its occurrence in the natural gas wells where helium is harvested, forcing gas suppliers to specify a lower bound for impurity levels at about $100 \mathrm{ppb}$ even in high-grade helium. In this paper, we present a simple apparatus to detect hydrogen traces present in liquid helium and easily check the quality of the liquid. Finally, we propose a solution to eliminate the hydrogen impurities in small- and large-scale helium recovery plants. The solution has been implemented in several laboratories that previously experienced a chronic occurrence of blocking, eliminating similar occurrences for more than one year.
\end{abstract}

DOI: 10.1103/PhysRevApplied.6.024017

\section{INTRODUCTION}

Liquid helium is the coldest fluid that exists in nature $(4.2 \mathrm{~K}$ at $100 \mathrm{kPa})$. It can be liquefied with the well-known commercial industrial liquefaction plants [large-scale helium recovery plants (LS HRPs)] derived from Collins technology [1] or with the more recently developed closedcycle refrigerator-based plants [small-scale helium recovery plants (SS HRPs)] [2-7]. Below its critical temperature $\left(T_{c}=5.2 \mathrm{~K}\right)$, any unwanted substance present in the liquid phase, i.e., any impurity, will be in solid form, resulting in

\footnotetext{
*Author to whom all correspondence should be addressed. crillo@unizar.es.

Published by the American Physical Society under the terms of the Creative Commons Attribution 3.0 License. Further distribution of this work must maintain attribution to the author(s) and the published article's title, journal citation, and DOI.
}

mist, snow, suspensions, or particulates. The vapor pressure of these solid impurities will be, in general, negligibly small $\left(\ll 10^{-9} \mathrm{~Pa}\right)$, except for the case of the hydrogen isotopes and their molecular combinations [8] for which the vapor pressure is of the order of $10^{-2}$ and $10^{-5} \mathrm{~Pa}$ at 5.2 and $4.2 \mathrm{~K}$, respectively. The solid impurities are usually charged and can be easily eliminated by electrostatic precipitation using Petryanov filters to obtain "optically clean" liquid, as demonstrated by Abrikosova and Shal'nikov [8]. But even optically clean filtered liquid helium may contain a significant quantity of nonsolid hydrogen, i.e., molecular hydrogen traces.

The $\mathrm{He}-\mathrm{H}_{2}$ gas mixture has attracted much interest in the scientific community because it is the simplest system for the study of intermolecular potentials [9-11]. The interaction potentials of hydrogen and helium have been extensively studied by Silvera [12]. The Lennard-Jones potential wells for the weakly interacting $\mathrm{He}-\mathrm{He}, \mathrm{He}-\mathrm{H}_{2}$, 
and $\mathrm{H}_{2}-\mathrm{H}_{2}$ pairs are $10.8,13.34$, and $34.3 \mathrm{~K}$, respectively. According to this study, $\mathrm{H}_{2}$ molecules may have a bound state with $\mathrm{He}$ atoms, reside in liquid-He surface states, and even penetrate into the liquid helium. Thus, in addition to the possible presence of hydrogen molecules in the helium vapor due to the non-negligible vapor pressure of solid hydrogen at $4.2 \mathrm{~K}$, there may exist also a non-negligible amount of these hydrogen molecules "dissolved" in the liquid-He phase.

Molecular $\mathrm{H}_{2}$ is naturally present in crude helium gas as extracted from natural gas sources [13]. In general, different methods are used to eliminate $\mathrm{H}_{2}$ prior to large-scale helium liquefaction for worldwide distribution [14,15]. However, in spite of the effort to eliminate it completely, very precise analytical methods indicate that even ultrahigh-purity commercial grade $\mathrm{He}$ gas, $99.9999 \%$ pure, thus, having less than $1000 \mathrm{ppb}$ in volume of total impurities, may contain up to $500 \mathrm{ppb}$ in volume of $\mathrm{H}_{2}$ (i.e., a hydrogen molar fraction $y_{\mathrm{H}_{2}}=5 \times 10^{-7}$ in He gas) [16-19].

It is also known that recovered gas in helium liquefaction plants may contain up to several parts per million in volume of $\mathrm{H}_{2}$ generated by oil degradation in compressors or pumps, outgassing of metallic pipes, or diffusion of naturally present atmospheric $\mathrm{H}_{2}$ [20] through plastic pipes and gas bags [21]. Thus, the presence of traces of $\mathrm{H}_{2}$ in a laboratory LS HRP and SS HRP up to the parts-per-million range $\left(y_{\mathrm{H}_{2}} \approx 10^{-6}\right)$ appears to be unavoidable.

In general, liquid helium in research laboratories is either delivered by a distributor of specialty gases or produced by liquefaction of both commercial grade and recovered gas. Liquid helium is subsequently stored and transferred to the application's cryostat requiring cryogenic cooling at atmospheric pressure and temperatures around $4.2 \mathrm{~K}$. Since the triple point of $\mathrm{H}_{2}$ is at $13.84 \mathrm{~K}$ and $7.04 \mathrm{kPa}$, the equilibrium vapor pressure of solid $\mathrm{H}_{2}$ at those temperatures (approximately $4.2 \mathrm{~K}$ ) is very small and of the order of approximately $10^{-5} \mathrm{~Pa}$. Therefore, if there is enough $\mathrm{H}_{2}$ in the He gas being liquefied to produce a partial pressure higher than the equilibrium vapor pressure at $4.2 \mathrm{~K}$, the $\mathrm{H}_{2}$ molecules will nucleate into solid clusters. At atmospheric pressure $\left(10^{5} \mathrm{~Pa}\right)$, those solid clusters will be in equilibrium with a $\mathrm{H}_{2}$ molar fraction in the vapor phase of the order of $10^{-10}\left[y_{\mathrm{H}_{2}} \approx\left(10^{-5} \mathrm{~Pa} / 10^{5} \mathrm{~Pa}\right)=10^{-10}\right]$.

Even though there are no experimental reports about the solubility of $\mathrm{H}_{2}$ in liquid helium, theoretical calculations from classical solubility theory [22] indicate that the limiting solubility of solid hydrogen in liquid helium at 4.2 K yields to molar fractions in the liquid phase $x_{\mathrm{H}_{2}}$ of the order of approximately $10^{-10}$, i.e., the same order of magnitude as the $\mathrm{H}_{2}$ molar fraction in the vapor phase $y_{\mathrm{H}_{2}}$.

Furthermore, the solid-hydrogen vapor pressure and the theoretical limiting solubility of solid hydrogen in liquid helium decrease exponentially with temperature, becoming both very small (approximately $10^{-9} \mathrm{~Pa}$ and $10^{-14}$, respectively) below $3 \mathrm{~K}$. Thus, the maximum concentration of $\mathrm{H}_{2}$ molecules present in liquid helium is determined by the exact temperature and pressure conditions of the helium bath. For the purpose of this work, the $\mathrm{H}_{2}$ molar fractions in the vapor $y_{\mathrm{H}_{2}}$ and in the liquid $x_{\mathrm{H}_{2}}$ below $3 \mathrm{~K}$, both being of the order of approximately $10^{-14}$ are considered negligible. Furthermore, at temperatures near or below $1 \mathrm{~K}$, hydrogen may be regarded as being completely insoluble in $\mathrm{He}$ [22].

Thus, unless $\mathrm{H}_{2}$ impurities are completely eliminated prior to He liquefaction, i.e., its molar fraction is reduced from its typical value in the range of $y_{\mathrm{H}_{2}} \approx 10^{-6}-10^{-5}$ down to approximately $10^{-14}$, the liquid $\mathrm{He}$ as produced contains traces of $\mathrm{H}_{2}$ up to a maximum concentration level determined by the temperature of the liquid (e.g. $x_{\mathrm{H}_{2}} \approx$ $10^{-10}$ at $4.2 \mathrm{~K}$ ). If the temperature of the liquid helium is further reduced, as it is the case in small capillary impedances for attaining very low temperatures $T<3 \mathrm{~K}$, the excess $\mathrm{H}_{2}$ will precipitate and accumulate at the impedance low-pressure side, and, after some time, it will produce a total impedance blockage.

A large number of applications requiring liquid-helium cooling are not sensitive to contaminants of any kind and, consequently, do not require special provisions for helium cleanliness and precautions to avoid contamination during liquid-helium refills. On the other hand, there is a considerable number of low-temperature applications that require achieving temperatures below $4 \mathrm{~K}$ [23] that are very sensitive to impurities present in the liquid, and, therefore, those applications need extreme-purity liquid helium for proper operation [24].

In Sec. II we present details of how $\mathrm{H}_{2}$ impurities give rise to the impedance-blocking problem. A plausible mechanism is proposed, and a detection tool for verifying the presence of molecular $\mathrm{H}_{2}$ in liquid helium is described. Section III deals with low-pressure $\mathrm{He}$ purification by cryocondensation and solutions for eliminating $\mathrm{H}_{2}$ impurities in SS HRPs. Lastly, in Sec. IV high-pressure He purification methods and solutions for eliminating $\mathrm{H}_{2}$ impurities in LS HRPs are given.

\section{LOW-TEMPERATURE IMPEDANCE BLOCKING}

\section{A. The impedance-blocking problem}

The impedance-blocking problem arises when liquid helium containing traces of $\mathrm{H}_{2}$ is transferred to a lowtemperature application or cryostat in which the liquid has to be pumped through a very small capillary or impedance tube to produce temperatures below $4 \mathrm{~K}$ using evaporation cooling, such as in magnetic-resonance-imaging systems [25]. In general, the impedance $Z$ is given in terms of the flow through the capillary $\dot{V}$ and the pressure drop across it $\Delta P$ (typically from 1 bar to 1 mbar) by 


$$
\dot{V}=\frac{\Delta P}{(\eta Z)}
$$

where $\eta$ is the viscosity of the liquid helium. Impedances in the range of $10^{10}$ to $10^{11} \mathrm{~cm}^{-3}$ are typically used in continuously pumping ${ }^{4} \mathrm{He}$ cryostats and inserts [26]. Cooling power of the order of $5 \mathrm{~mW}$ or more is easily achievable at the impedance outlet once we consider the heat of evaporation of ${ }^{4} \mathrm{He}$ (approximately equal to $83 \mathrm{~J} / \mathrm{mol}$ ) and a typical flow rate of $\dot{V}=10^{-4} \mathrm{~mol} / \mathrm{s}$ that can be obtained with a midsized mechanical pump. In practice, the impedance is built with an appropriate length of fine CuNi capillary tube, usually with a short length of wire inside to increase the impedance value.

In an attempt to reduce any impedance blocking, a widespread and generally accepted "low-temperature best practice" is to stop any solid impurities at strategic locations along the helium supply chain with submicron metal sintered filters (e.g., Mott Corporation). The first opportunity to do so in a typical laboratory workflow is while transferring helium from a storage Dewar into the application cryostat for the first time. For this purpose, many laboratories incorporate a metal filter at the outlet "tip" of the helium transfer line so that the solid impurities are trapped in the line and not transferred into the application cryostat. Once the helium transfer is complete, the line is warmed up, the impurities are flushed away, and the tip ultrasonically cleaned. If any impurities should make it past the first filter, and, furthermore, to filter any other solid impurities already present in the application apparatus, a second "best practice" employed by cryostat designers is to incorporate a similar type of filter at the inlet of the impedance tube at the cold end of the apparatus cryostat.

It is important to appreciate, however, that mechanical filtering of this type is limited in its effectiveness and cannot selectively discriminate and separate $\mathrm{H}_{2}$ molecules from their helium carrier flow (a two-phase liquid- and vapor helium flow), neither during liquid transfer nor during pumping. This is because in spite of the relatively high binding energies reported for hydrogen with the surface of some solids [27,28], which involve potentials of the order of several hundred kelvin, the specific surface area per unit volume of the metal sintered filters commonly used in this application (see, for example, Mott $316 \mathrm{~L}$ ) is below $0.5 \mathrm{~m}^{2} / \mathrm{g}$. This is 3 to 4 orders of magnitude smaller than the area per unit volume exhibited by state-of-the-art solid- $\mathrm{H}_{2}$ storage devices. Moreover, the porous size of the media grade selected $(0.5 \mu \mathrm{m})$ is also more than 3 orders of magnitude larger than the $\mathrm{H}_{2}$ molecular radius. On the basis of these considerations, the contribution of $\mathrm{H}_{2}$ physical sorption on the walls of the mechanical filter is estimated to be very small and, furthermore, limited by the very small vapor pressure of solid hydrogen at the temperature of liquid $\mathrm{He}$.
Thus, in light of these considerations, we postulate that despite the adoption of these simple best practices, $\mathrm{H}_{2}$ molecules will inevitably enter first the application cryostat during helium refills and then the impedance fine-capillary tubes during continuous operation below $4 \mathrm{~K}$. As a result, part of the $\mathrm{H}_{2}$ molecules carried by the helium flow will freeze (or precipitate) inside the capillary. This is a consequence of the reduction in temperature and total pressure of helium, which is accompanied by a sizable reduction in solid-hydrogen vapor pressure and in the hydrogen-limiting solubility in liquid helium, so that sooner or later depending on the specific dimensions and helium flow rate pumped through the capillary, a blockage will appear. When this happens, the whole setup has to be warmed up, at least up to about $14 \mathrm{~K}$ (hydrogen melting point) but more often to room temperature, with a dramatic loss of time and cost of helium.

\section{B. The impedance-blocking mechanism}

Figure 1 illustrates molecular $\mathrm{H}_{2}$ present in the liquidhelium bath flowing through a submicron-sized metallic sintered filter (for example, with a 500-nm average pore size) placed to stop solid impurities from entering the finecapillary impedance tube. When the temperature in the capillary is reduced below $3 \mathrm{~K}$ by evaporation cooling, the $\mathrm{H}_{2}$ vapor pressure, as well as the limiting solubility of $\mathrm{H}_{2}$ in helium, becomes negligibly small $\left(y_{\mathrm{H}_{2}}, x_{\mathrm{H}_{2}}<10^{-14}\right)$. Therefore, as it will be quantitatively calculated in Sec. III A, all the $\mathrm{H}_{2}$ present in the liquid helium heterogeneously nucleates along the walls of the impedance tube.

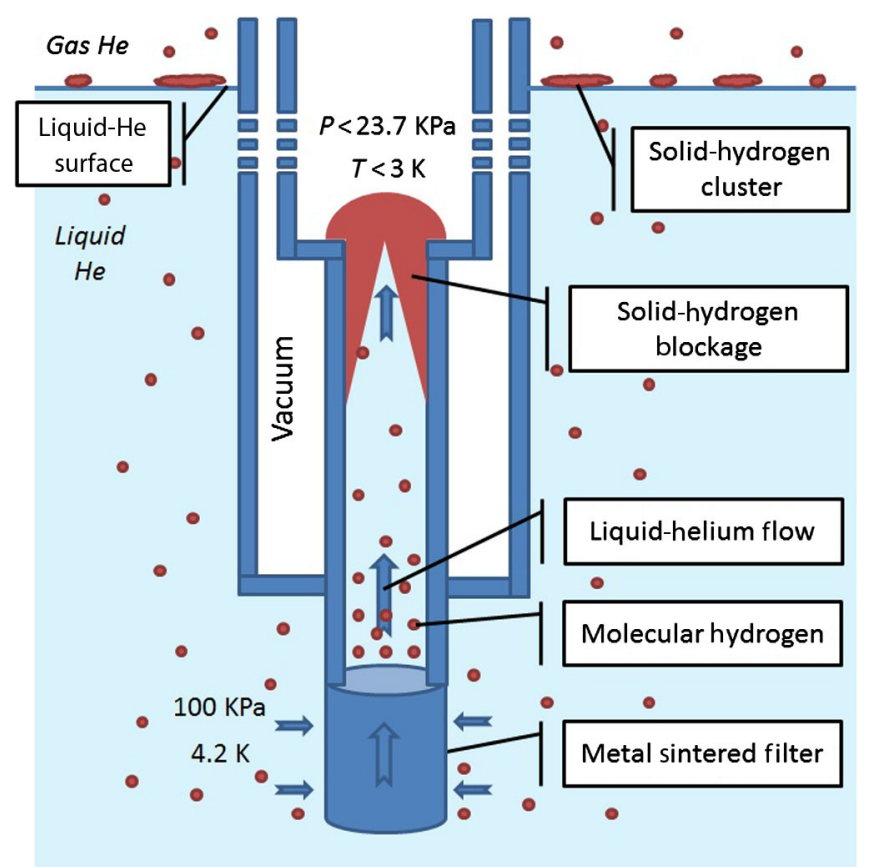

FIG. 1. Schematic description of low-temperature impedance blockage by molecular $\mathrm{H}_{2}$ present in liquid He. 
A similar mechanism for the freezing of water molecule impurities in nitrogen gas has been proposed to explain blocking in micromachined Joule-Thomson coolers operating at approximately $100 \mathrm{~K}[29,30]$.

As an example, a typically two-phase He flow of only 1 standard liter per minute (SLPM) having $x_{\mathrm{H}_{2}}=0.35 \mathrm{ppb}$ $\left(3.5 \times 10^{-10}\right)$ of $\mathrm{H}_{2}$ molecules [i.e., corresponding to the vapor pressure of solid hydrogen in liquid helium under typical laboratory conditions $\left(4.2 \mathrm{~K}\right.$ and $\left.10^{5} \mathrm{~Pa}\right)$ ] pumped through a cylindrical tube impedance of $66-\mu \mathrm{m}$ effective diameter [e.g., the low- $T$ impedance of a Quantum Design physical property measurement system (PPMS)] [31] may produce a solid-hydrogen cylindrical block of $66 \mu \mathrm{m}$ diameter that in about $24 \mathrm{~h}$ will have $132 \mu \mathrm{m}$ height. The exact time for the blocking to occur will depend on the exact solid-hydrogen distribution in the impedance. Instead, several years are necessary to produce the same effect when pumping helium with a lower concentration of $\mathrm{H}_{2}$ molecules like that corresponding to the vapor pressure of solid hydrogen at $3 \mathrm{~K}, x_{\mathrm{H}_{2}}=0.0075 \mathrm{ppt}\left(7.5 \times 10^{-15}\right)$. This experimental fact is the reason why we consider the vapor pressure of solid hydrogen at $3 \mathrm{~K}$ negligibly small regarding impedance blockage.

\section{Experimental apparatus for $\mathbf{H}_{2}$-blocking detection}

To experimentally verify the validity of the proposed blocking mechanism, an apparatus containing a "test impedance" is built (see Fig. 2). It consists of a liquidhelium transfer line adapted to receive a thin-capillarybased impedance at one of its ends. The impedance is made of a $150-\mu \mathrm{m}$-inner-diameter stainless-steel capillary tube fitted with a $135-\mu$ m-outer-diameter copper/niobium filament inside of it. With the purpose of reducing the blocking time as much as possible, a constriction is created in the capillary tube by mechanically "pinching it" at a location along its length. By pumping liquid helium with a scroll pump connected at the other end of the line, a temperature reduction of the cryogenic fluid flowing through the

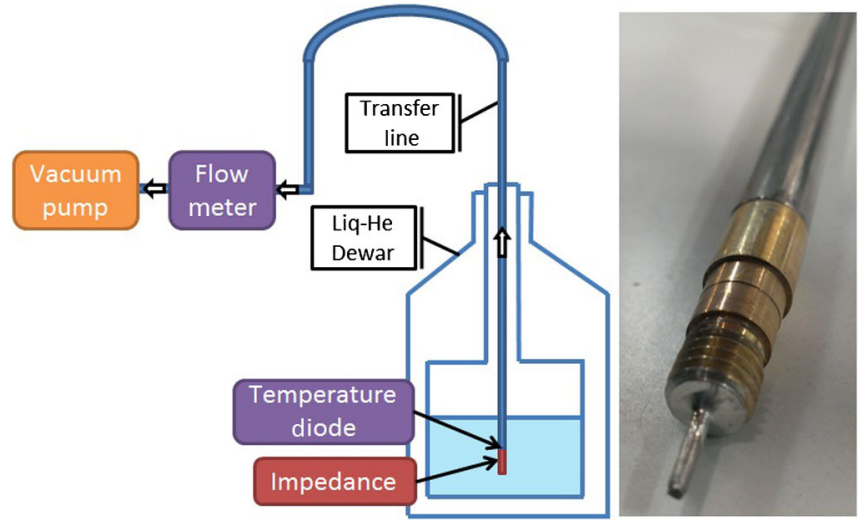

FIG. 2. Left: Schematic setup of the hydrogen detection device. Right: Photograph of the impedance at the end of a transfer line. impedance is achieved. This temperature is measured with a calibrated diode DT-670-SD from Lake Shore. Moreover, a mass flow and pressure meter (implemented with model GSB-B9TA-BB26 from Vögtlin Instruments) is used to measure the He mass flow through the impedance and the pressure at its output.

The apparatus is inserted inside a commercial liquid-He transport Dewar. Pumping He through the apparatus produces a temperature reduction from 4.2 to below $3 \mathrm{~K}$ as read by the diode thermometer located near the impedance. At this temperature, the solid-hydrogen vapor pressure and its limiting solubility in liquid helium are negligibly small; thus, all the molecular hydrogen passing through the impedance is solidified. The data in Fig. 3 (top) show that in about $10 \mathrm{~h}$ a complete blockage in the impedance appears, and then the temperature increases to $4.2 \mathrm{~K}$, while the $\mathrm{He}$ flow decreases to zero. To demonstrate unambiguously that the $\mathrm{H}_{2}$ molecules are responsible of the blockage, the apparatus is moved up along the Dewar neck until the temperature reading of the diode reaches $15 \mathrm{~K}$ just above the hydrogen normal melting point $(14 \mathrm{~K})$. Then, it is again introduced in the liquid $\mathrm{He}$ with a consequent recovery of gas flow and a reduction of temperature below $3 \mathrm{~K}$, as shown in Fig. 3 (bottom). This confirms that solid $\mathrm{H}_{2}$ is the most likely candidate for the
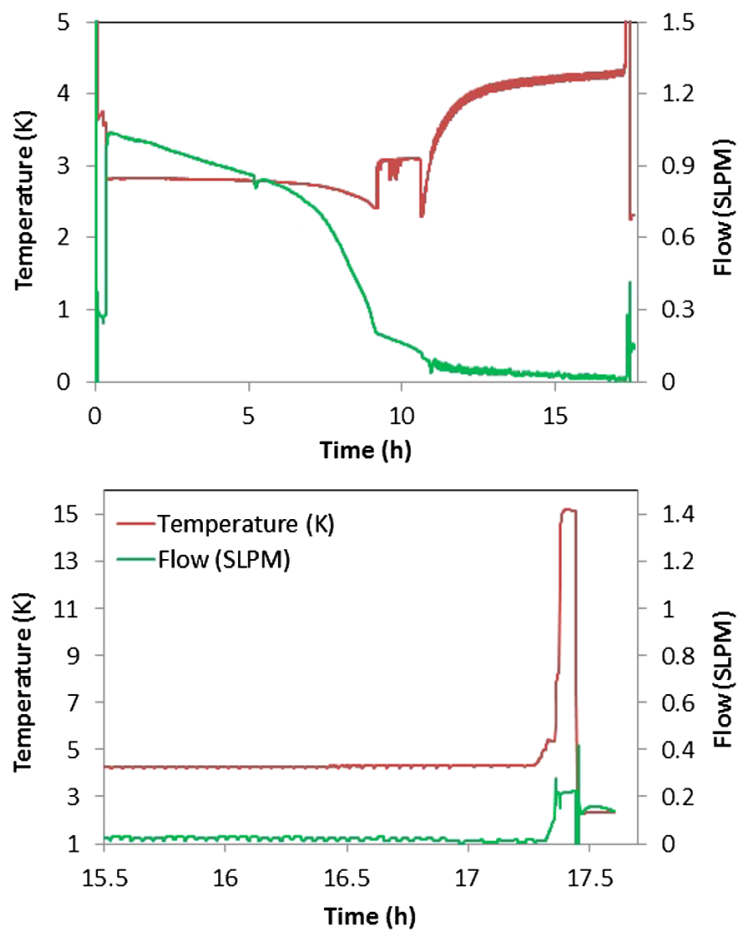

FIG. 3. Top: Temperature and gas flow measured with the test impedance during the blocking process. The blockage occurs after about $10 \mathrm{~h}$. Bottom: Detail of the impedance unblocking. The impedance is heated to about $15 \mathrm{~K}$ by moving it up in the Dewar neck where the flow is partially reestablished and, then, is cooled again. 


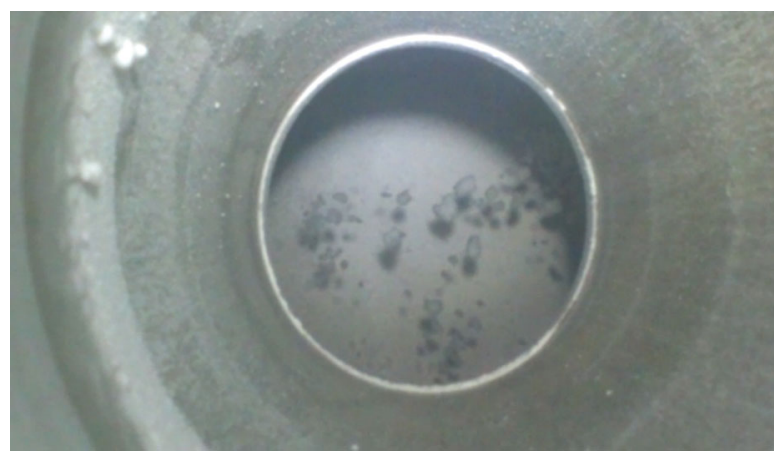

FIG. 4. Top view of a liquid-helium Dewar through a methacrylate cover. The hydrogen clusters project a shadow on the Dewar bottom.

observed blocking-unblocking results under the above conditions.

The setup and the experimental data are shown in Figs. 2 and 3 , respectively. It is worthwhile mentioning that no blockage at all is observed when liquid helium is just circulated (i.e., with no pressure drop) through a metal sintered filter alone, thus, confirming that $\mathrm{H}_{2}$ physisorption on the sintered filter walls is negligible in this configuration. This behavior is indeed analogous to what occurs in commercial cryostats such as the PPMS. In these systems, the precise control of the sample temperature is flawless, even when $\mathrm{H}_{2}$ is present in the helium bath as long as the temperature at the outlet of the impedance is maintained above $4.2 \mathrm{~K}$. However, when a temperature below $4.2 \mathrm{~K}$ is set and the system starts pumping on the flow impedance creating a pressure drop across it, the flow will plug within $24 \mathrm{~h}$.

The direct visualization is definitive proof of the hydrogen presence in liquid He. For this purpose, we use a transparent methacrylate cover on the top of a liquidhelium Dewar having a wide neck. Using this setup, it is possible then to directly view the liquid-He surface from the top. When the hydrogen concentration in the Dewar is higher than that corresponding to the vapor pressure of $\mathrm{H}_{2}$ at the boiling point of $\mathrm{He}$, solid "clusters" nucleate. Since hydrogen is the only solid element with a density lower than that of liquid $\mathrm{He}(80 \mathrm{vs} 125 \mathrm{~g} / \mathrm{L}$ ), these solid clusters float on the surface of the liquid and project a shadow on the Dewar bottom so that they are seen very clearly (see Fig. 4 and Video 1).

\section{SOLUTION FOR SMALL-SCALE HELIUM RECOVERY PLANTS: THE LOW-PRESSURE GREEN HELIUM PLANT CONCEPT}

As explained in Sec. II, in order to eliminate the impedance-blockage problem, the hydrogen concentration in helium has to be reduced from the typical values found in commercial or in recovered gas, that is, $y_{\mathrm{H}_{2}} \approx 10^{-7}-10^{-6}$, down to molar fractions of the order of $y_{\mathrm{H}_{2}}, x_{\mathrm{H}_{2}} \approx 10^{-14}$ in

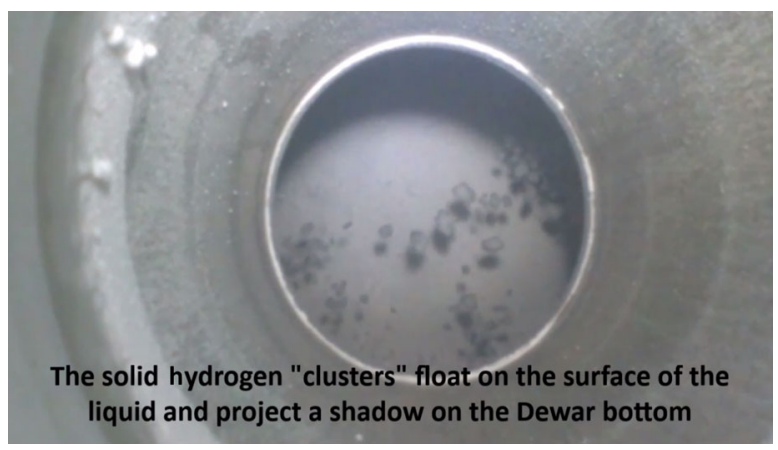

VIDEO 1. The video shows floating hydrogen clusters in liquid helium.

the liquid and in the vapor phases. Such very low hydrogen concentrations can be attained by (i) lowering the temperature of the gas down to $3 \mathrm{~K}$ using a cryocondensation purification process (see Sec. III A) (as we explain in Sec. III C, this is feasible only for flow rates below 10 SLPM) or (ii) circulating the gas through a nonevaporable getter-based in-line purifier.

However, for process (ii) to be effective, the input gas of the in-line purifier should be free of any other impurity different from $\mathrm{H}_{2}$, otherwise, the total sorption capacity of $\mathrm{H}_{2}$ is reduced by the reactions of the getter material with $\mathrm{O}_{2}, \mathrm{~N}_{2}, \mathrm{H}_{2} \mathrm{O}$, and other impurities.

Thus, as explained in detail in what follows, the combination of processes (i) and (ii) appears to be the optimum solution for any flow rate and any impurity concentration level.

\section{A. Purification of He by cryocondensation}

In general, purification by cryocondensation is accomplished by causing a phase change of the impurities sought to be removed from the gas to be purified. The effectiveness of this method depends upon the vapor pressure of the impurities at the purification temperature.

When a mixture of helium gas and its typical impurities with total constant pressure $p_{t}$ is cooled down for purification using cryocondensation, the partial pressure of any impurity in the vapor phase $p_{i}(T)$ remains constant with $T$ until the condensation saturation line of the impurity is reached. This change in partial pressure occurs at a temperature $T_{i}$ that depends on the initial molar fraction $y_{i}(300 \mathrm{~K})$. In other words, above $T_{i}$, the $p_{i}(T)$ values can be approximated by the product of the initial molar fraction in the vapor phase and the total gas pressure [32]:

$$
p_{i}(T)=y_{i}(300 \mathrm{~K}) P_{t}, \quad T>T_{i} .
$$

Once the corresponding vapor-pressure saturation line of the impurity is reached at $T_{i}$, the partial pressure of the impurity in the vapor phase coincides with its vapor pressure $\pi_{i}(T)$, 


$$
p_{i}(T)=\pi_{i}(T), \quad T \leq T_{i}
$$

Therefore, once the condensation of a given impurity starts, its molar fraction in the vapor phase is not constant anymore but decreases dramatically with $T$, as $\pi_{i}(T)$ also does, and is given by

$$
y_{i}(T)=\frac{\pi_{i}(T)}{p_{t}}, \quad T \leq T_{i}
$$

Figure 5 shows the molar fraction variation $y_{i}(T)$ of $\mathrm{O}_{2}$ and $\mathrm{H}_{2}$ calculated from Eqs. (2) and (4), having initial molar concentrations of $10^{-4}$ and $10^{-7}$, respectively, for a helium-gas purification pressure $p_{t}=10^{5} \mathrm{~Pa}$. For clarity, the figure also includes the $P$ - $T$ liquid-vapor saturation line for the condensed phase of the carrier gas, $\mathrm{He}$ (I), and the $P-T$ liquid-vapor and solid-vapor saturation lines for the most frequent impurities typically found in recovered helium, from 3 up to $300 \mathrm{~K}$. The critical point of $\mathrm{He}$ (I) and the triple points of the impurities are marked.

Thus, from a simple inspection of Fig. 5 we conclude that below $20 \mathrm{~K}$, only $\mathrm{Ne}$ and $\mathrm{H}_{2}$ may have a non-negligible gas molar fraction in He. Furthermore, below $6 \mathrm{~K}$, only $\mathrm{H}_{2}$ gas can flow together with liquid helium after mechanical filtering.

In fact, the presence of $\mathrm{O}_{2}$ and $\mathrm{N}_{2}$ molecules in helium can be neglected below 24 and $20 \mathrm{~K}$, respectively. However, the presence of $\mathrm{H}_{2}$ molecules cannot be neglected until temperatures well below $3 \mathrm{~K}$ are reached.

Thus, to obtain ultrapure liquid helium using this purification method, i.e., to get liquid $\mathrm{He}$ free of any relevant molar fraction of any impurity and, more specifically, free of molecular $\mathrm{H}_{2}$ (that we will call "green helium" in what follows), the He temperature shall be reduced down to at least, $3 \mathrm{~K}$.

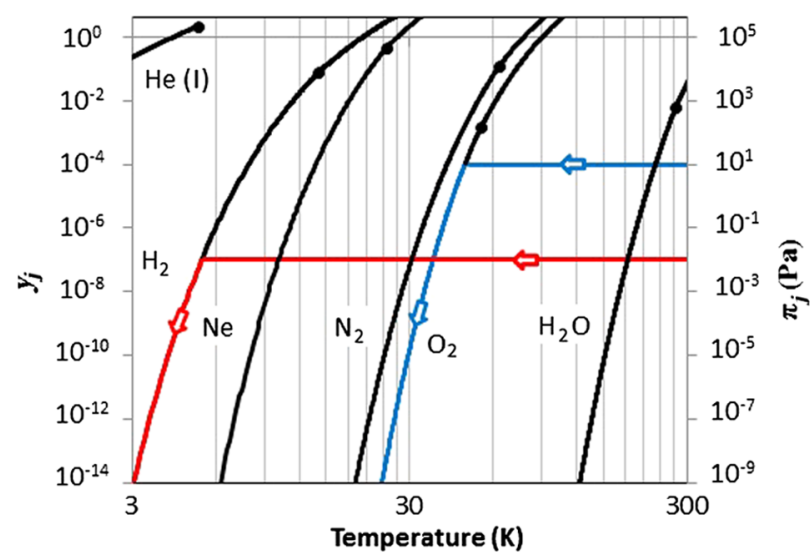

FIG. 5. Molar fractions $y_{i}(T)$ and partial pressures $p_{i}(T)$ of $\mathrm{O}_{2}$ (blue) and $\mathrm{H}_{2}$ (red) in recovered helium as a function of $T$, for initial molar fractions of $10^{-4}$ and $10^{-7}$, respectively, at STP $\left(10^{5} \mathrm{~Pa}\right.$ and $\left.300 \mathrm{~K}\right)$. Black solid lines are the equilibrium vapor pressure $P-T$ line of $\mathrm{He}(\mathrm{I})$ and the vapor-pressure lines of some typical impurities $\pi_{i}(T)$ as a function of $T$ [33], in the range $3-300 \mathrm{~K}$.

\section{B. Pumping two-phase liquid-vapor He through a capillary impedance tube}

When two-phase liquid-vapor $\mathrm{He}$ is pumped from a bath at $4.2 \mathrm{~K}$ and $10^{5} \mathrm{~Pa}$ through a capillary impedance tube, the He stream cools down through its $P-T$ vapor-liquid equilibrium saturation line, $\pi_{\mathrm{He}}(T)$ (see Fig. 5). Thus, if there is enough hydrogen to form solid clusters, the saturation molar fraction of molecular $\mathrm{H}_{2}$ in the vapor phase will be the starting concentration, $\left.y_{\mathrm{H}_{2}}(T)\right|_{\mathrm{eq}}$. This value can be calculated using the vapor-pressure saturation line of hydrogen $\pi_{\mathrm{H}_{2}}(T)$ by Eq. (4),

$$
\left.y_{\mathrm{H}_{2}}(T)\right|_{\mathrm{eq}}=\frac{\pi_{\mathrm{H}_{2}}(T)}{\pi_{\mathrm{He}}(T)}, \quad T \leq 4.2 \mathrm{~K}
$$

On the other hand, at the very low concentration levels under discussion, solid hydrogen may dissolve in the liquid [22]. In that case, the molar fraction of solid $\mathrm{H}_{2}$ dissolves in the liquid phase $x_{\mathrm{H}_{2}}(T)$ and may be estimated from classical solubility theory.

Figure 6 shows the saturation molar fraction of $\mathrm{H}_{2}$ in the vapor phase $\left.y_{\mathrm{H}_{2}}(T)\right|_{\mathrm{eq}}$ calculated using expression (5) in the interval 3-4.2 K (solid line). Similarly, the molar fraction of $\mathrm{H}_{2}$ obtained from its theoretical limiting solubility in the liquid phase $\left.x_{\mathrm{H}_{2}}(T)\right|_{\mathrm{eq}}$ obtained from expression (1) in the work of Jewel and McClintock [22] (dashed line) is also shown. Both are very similar.

Thus, a well-defined lower limit for $\mathrm{H}_{2}$ concentration as a function of the temperature in the helium-vapor phase is obtained from the vapor pressure of solid hydrogen. Since solid hydrogen can be considered as a volatile solute (i.e., the solute vapor pressure is not negligible) for $T>3 \mathrm{~K}$, there is a well-defined minimum solubility in the liquid phase for each temperature. This minimum solubility is

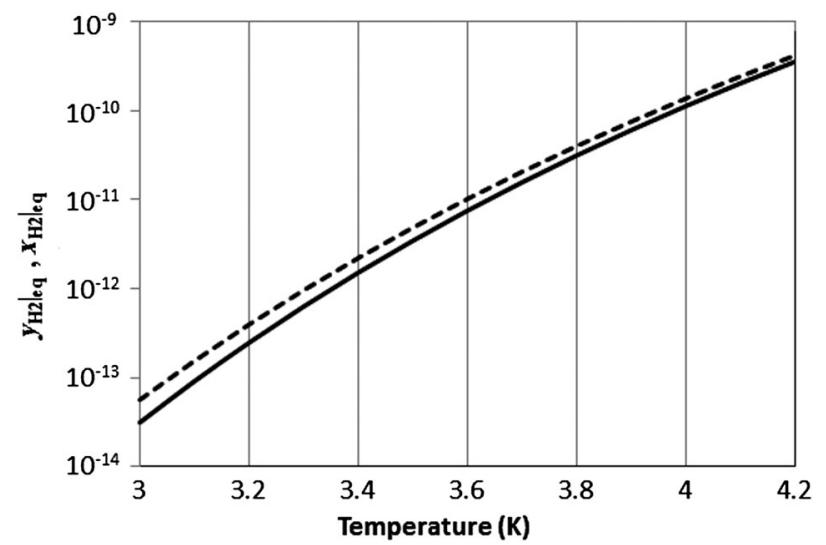

FIG. 6. Low-temperature $\mathrm{H}_{2}$-saturation molar fraction in helium obtained from the limiting solubility of $\mathrm{H}_{2}$ in $\mathrm{He}$ [dashed line, $\left.\left.x_{\mathrm{H}_{2}}(T)\right|_{\mathrm{eq}}[22]\right]$ and from the $\mathrm{H}_{2}$ equilibrium-saturation vapor pressure [solid line, $\left.y_{\mathrm{H}_{2}}(T)\right|_{\mathrm{eq}}[33]$ ], as a function of $T$ in the range $3-4.2 \mathrm{~K}$ at $\pi_{\mathrm{He}}(T)$. 
also obtained from the vapor pressure. Furthermore, to know whether the actual value of the solubility of $\mathrm{H}_{2}$ in the liquid phase is higher than the minimum value is not relevant because this already justifies the experimentally observed blockage times. In fact, if it is higher, it will just reduce the blockage time of the impedance.

Thus, when the pumped helium stream expands and cools down inside the capillary impedance from 4.2 to $3 \mathrm{~K}$, the $\mathrm{H}_{2}$ molar fraction in the two-phase liquid-vapor helium $\left(\left.x_{\mathrm{H}_{2}}(T)\right|_{\mathrm{eq}},\left.y_{\mathrm{H}_{2}}(T)\right|_{\mathrm{eq}}\right)$ flowing through the impedance decreases by 4 orders of magnitude (from approximately $10^{-10}$ to approximately $10^{-14}$ ), and, consequently, the excess $\mathrm{H}_{2}$ freezes or precipitates and blocks the capillary.

\section{The solution for low-pressure $\left(P<P_{c}\right)$ SS HRP based on smart advanced technology purifiers and advanced technology liquefiers}

Our green helium (extreme purity $\mathrm{He}$ free of molecular $\left.\mathrm{H}_{2}\right)$ low-pressure $\left(P<P_{c}\right)$ SS HRP concept is depicted in Fig. 7. The plant is initially fed commercial grade $99.999 \%$ pure helium gas that may contain up to $\mathrm{a}_{2}$ molar fraction of $10^{-6}$ [14] ("red helium"). The gas is further purified by cryocondensation by one or more cryo-refrigerator-based advanced technology purifiers (ATPs) [2] each with a total effective volume to store solid impurities of several liters and a maximum purification flow rate of around 10 SLPM at $3 \mathrm{~K}$ and $30 \mathrm{SLPM}$ at $20 \mathrm{~K}$. The special prototype of ATP30 [2] used in this work traps $\mathrm{H}_{2}$ and any other impurity present in the gas by lowering the temperature of the input gas stream down to $T<3 \mathrm{~K}$, at a pressure of $240 \mathrm{kPa}$, just slightly above the critical pressure of $\mathrm{He}$. At
$T<3 \mathrm{~K}$, all the $\mathrm{H}_{2}$ molecules are trapped inside the purifier, since its residual vapor pressure becomes negligibly small $\left(<10^{-9} \mathrm{~Pa}\right)$.

The $\mathrm{H}_{2}$-free $\mathrm{He}$ from the ATPs is then fed through the bypass, a parallel ensemble of advanced technology liquefiers (ATLs) $[2,4]$ that produce $\mathrm{H}_{2}$-free ("green") ultrapure liquid helium. The instruments are always filled with ATL green liquid helium. Obviously, commercial liquid helium should never be transferred to hydrogen-sensitive instruments unless the absence of $\mathrm{H}_{2}$ is first verified using a test apparatus like the one described in Sec. II C (e.g., gas-flow measurement through the test impedance remains constant during several hours). In this helium recovery plant, helium boiloff from the cryogenic instruments is collected in a gasbag and compressed in the recovery bottles at $20 \mathrm{MPa}$. $\mathrm{A}_{2} \mathrm{O}$ dryer plumbed in series after the compressor, not shown in the scheme of Fig. 7, should always be used.

When a pressure drop develops between the input and the output of one of the ATPs due to the accumulation of solid impurities $\left(\mathrm{H}_{2}, \mathrm{~N}_{2}, \mathrm{O}_{2}\right)$, an ATP regeneration process is automatically initiated (see Fig. 8). The input and the output gas ports of the given ATP are closed, so that this ATP is now isolated, and the entire ATP Dewar volume is heated up to around $130 \mathrm{~K}$ so that all the low-vaporpressure impurities collected in solid form, e.g., $\mathrm{H}_{2}, \mathrm{~N}_{2}$, and $\mathrm{O}_{2}$, are sublimated and released into the atmosphere through a vent valve. Before restarting a new purification cycle, the ATP cools down again to the temperature of normal operation at $3 \mathrm{~K}$.

In this way, $\mathrm{H}_{2}$ molecules are completely eliminated, but the ATP input flow is restricted by the available coldhead cooling capacity at $3 \mathrm{~K}$, e.g., around 10 SLPM for

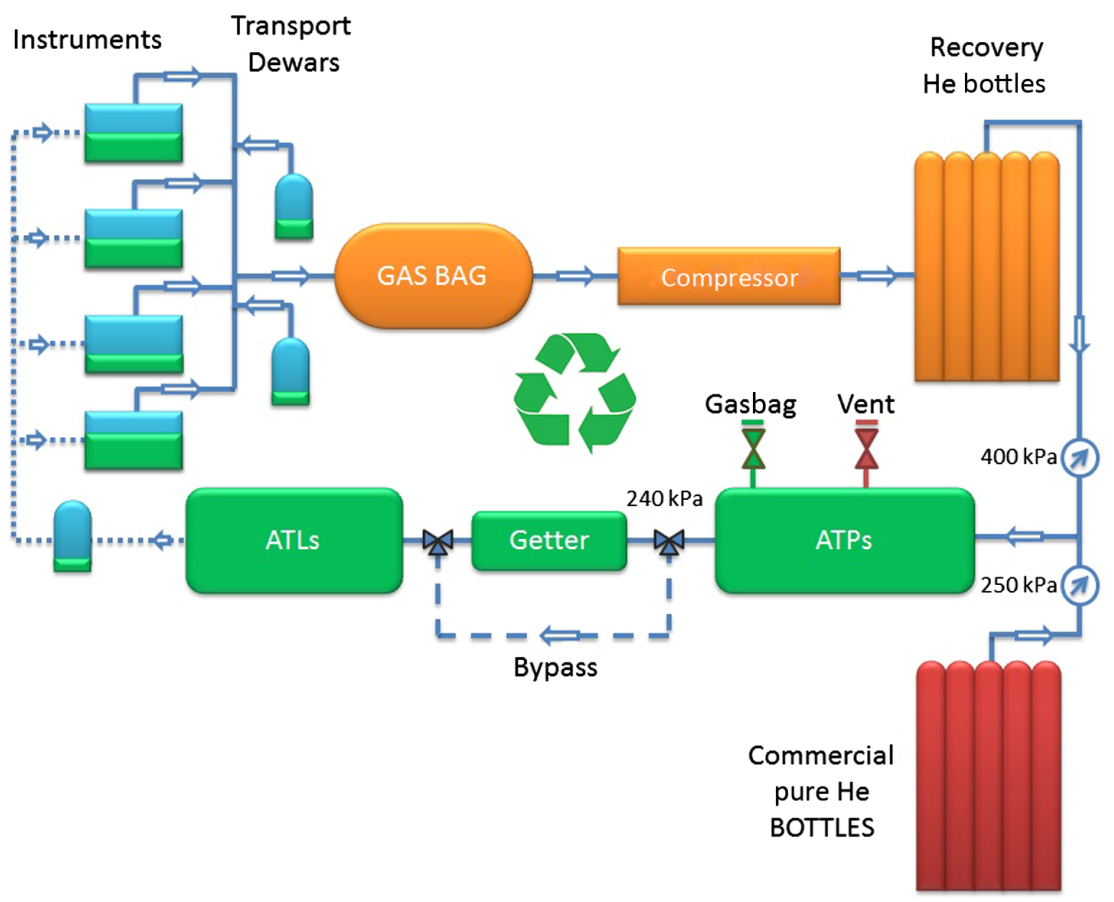

FIG. 7. Schematic configuration of a small-scale low-pressure $(<250 \mathrm{kPa})$ green helium recovery plant (free of hydrogen). Gasbag, compressor, and recovery helium bottles are not completely free of $\mathrm{H}_{2}$ (orange). The commercial $\mathrm{He}$ bottles are the main source of contamination (red). The bypass is closed when the ATP operation temperature is $T>3 \mathrm{~K}$. 


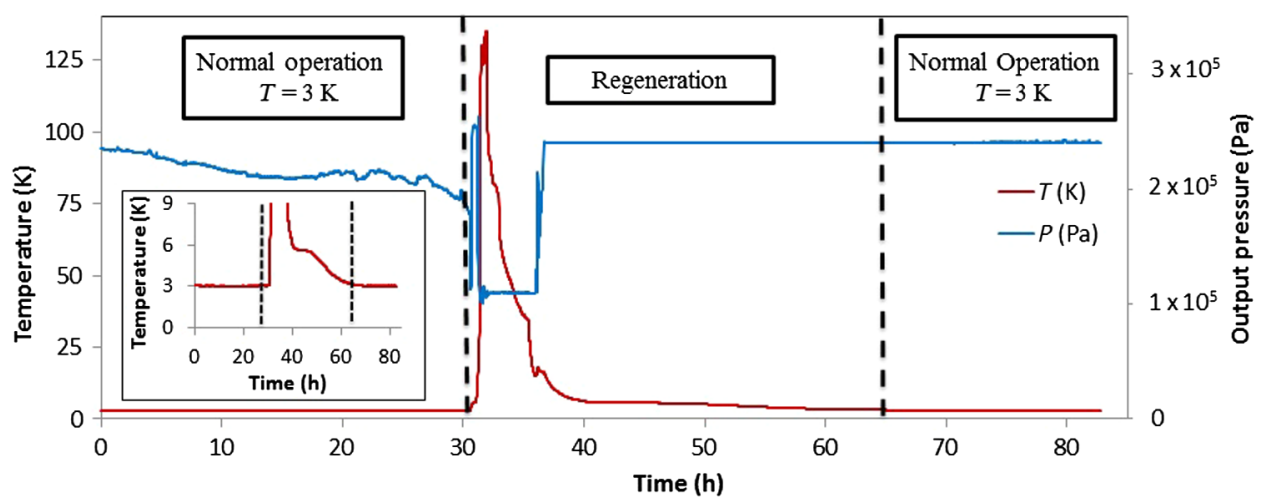

FIG. 8. ATP output pressure and temperature during regeneration. Purification temperature remains around $3 \mathrm{~K}$ in normal operation and reaches around $130 \mathrm{~K}$ during a regeneration process. Then, after releasing all the collected impurities into the atmosphere, the purifier returns to a normal operation (240 kPa, $3 \mathrm{~K})$.

a 1-W class Gifford-McMahon two-stage cryorefrigerator (Sumitomo RDK-408), and, in addition, a small amount of He (around $1 \%$ on average) is lost during each regeneration cycle.

For higher input flows from 10 up to 30 SLPM, the purification temperature is in the range between 5 and $20 \mathrm{~K}$, and this does not guarantee a negligible vapor pressure of solid $\mathrm{H}_{2}$ or a negligible solubility in liquid He. Thus, the purified gas will still contain $\mathrm{H}_{2}$ molecules that need to be eliminated before liquefaction. A solution tested in this work consists of the chemisorption of the remaining $\mathrm{H}_{2}$ molecules in the ATP output gas by a getter material at room temperature. The nonevaporable getter materials used in this work are (i) $[\mathrm{Zr}(70 \%) \mathrm{V}(24.6 \%$, Fe (5.4\%)]-based alloy working at room temperature and (ii) [Ni (31\%) $\mathrm{NiO}$ (32\%) $\mathrm{SiO}_{2}(24 \%) \mathrm{MgO}(13 \%)$ ]-based oxides working at room temperature (Quantum Design part no. HM1172).

At high flow rates, the ATP loses efficiency long before a pressure drop appears. The ATP includes the so-called "soft-regeneration" process (see Fig. 9), during which only the cold-head region of the ATP is warmed above $80 \mathrm{~K}$, while the output filter is kept below $25 \mathrm{~K}$ so that all the trapped $\mathrm{H}_{2}$ molecules in solid form are now sublimated and captured by the getter. The solid $\mathrm{N}_{2}$ and $\mathrm{O}_{2}$ still have a negligible vapor pressure below $10^{-9} \mathrm{~Pa}$ (see Fig. 5) and

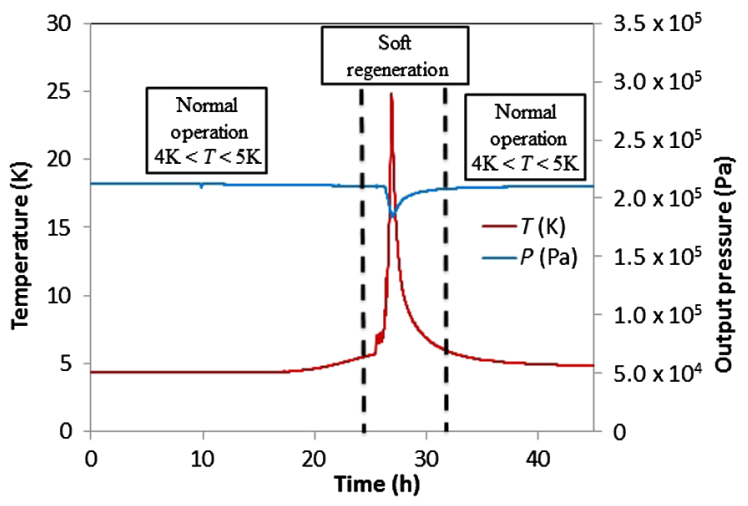

FIG. 9. ATP purification temperature remains around $4 \mathrm{~K}$ in normal operation and reaches around $25 \mathrm{~K}$ during a softregeneration process. At this moment, a great quantity of hydrogen accumulated is released and is trapped by the getter material. Then the purifier returns to a normal operation. remain frozen in the ATP till a regeneration due to a pressure drop is needed.

During soft-regeneration processes, the ATP continues feeding the ATL liquefiers through the getter. This solution is extremely efficient since there are no helium losses at all. On the other hand, in this configuration, the getter traps only hydrogen and it does so in a reversible way. Therefore, once it is near saturation, typically every two years, it can be regenerated by heating it to a specific $\mathrm{H}_{2}$ desorption temperature (typically, $>500{ }^{\circ} \mathrm{C}$ ).

When a getter material is used, the purification temperature can be in the range of $10-30 \mathrm{~K}$, and the ATP can use a 10-K class cryorefrigerator (i.e., Sumitomo CH-208 R) that is more economical than a $4-\mathrm{K}$ class cryorefrigerator.

This green helium gas at the getter output is eventually liquefied in a commercial ATL and transferred directly or by intermediate transport Dewars into the application instruments. The evaporated gas from non- $\mathrm{H}_{2}$-sensitive instruments that could be initially filled with commercial "no-green" liquid (e.g., NMRs, Magnetoencephalographs, high-field magnet cryostats, etc.) and can have a hydrogen quantity equal to or below that corresponding to the vapor pressure of the hydrogen at $4.2 \mathrm{~K}$ and $10^{5} \mathrm{~Pa}$ (i.e., $\left.y_{\mathrm{H}_{2}}=3.5 \times 10^{-10}\right)$ is also collected in the gasbag, compressed, and injected again in the ATPs for purification and complete elimination of the $\mathrm{H}_{2}$ impurities.

The validity of the green helium plant concept is demonstrated by the fact that impedance blockages are completely eliminated for more than one year when the plant configuration is implemented in the Cryogenic Liquids Service at the University of Zaragoza. Furthermore, the efficiency of the thermally activated getter media in trapping $\mathrm{H}_{2}$ is verified by the continuous in-line $\mathrm{H}_{2}$ monitoring described below. The graphs in Figs. 10(a) and 10(b) show the filter temperature of the ATP special prototype as a function of time. During the test, this temperature is varied from the base temperature of the purifier up to about $25 \mathrm{~K}$ and back to the base temperature in about $2.5 \mathrm{~h}$. The hydrogen concentration in the heliumpurified carrier gas is monitored using a mass spectrometer (helium leak detector QualyTest HLT260 from Pfeiffer Vacuum with hydrogen mass selected). During the test, the 
solid $\mathrm{H}_{2}$ collected in the ATP sublimates. The getter efficacy capturing $\mathrm{H}_{2}$ molecules is demonstrated by placing the $\mathrm{H}_{2}$ detector to analyze the helium gas at the output of the ATP [see Fig. 10(a)] and at the output of the heated getter [see Fig. 10(b)], respectively.

The hydrogen concentration data $\left[\mathrm{H}_{2}(t)\right]$ obtained with the above technique can be represented as a function of the ATP output filter temperature $T(t)$ for a proper understanding. Figure 11 shows the $\left[\mathrm{H}_{2}(T)\right]$ concentration peak in arbitrary units (red circles) observed at the ATP output after a period of a six days purifying recovered helium of a SS HRP recovery plant without the getter element. The green circles correspond to data taken in the same SS HRP plant at the output of the getter after five days purifying recovered helium gas (of the order of $10^{5}$ standard liters in both cases). Clearly, the $\mathrm{H}_{2}$ concentration is drastically reduced below the hydrogen detector background level, from 107 to less than 2 arb. units. Thus, the green points correspond to data taken in a hydrogen-free green helium recovery plant.
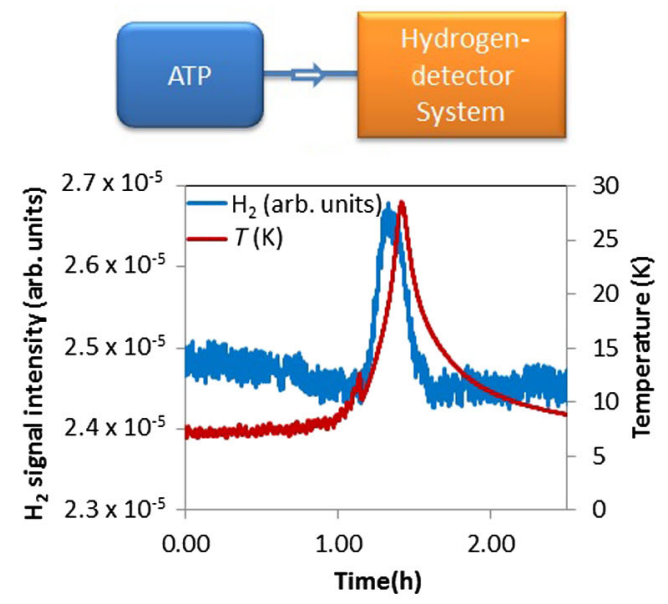

(a)
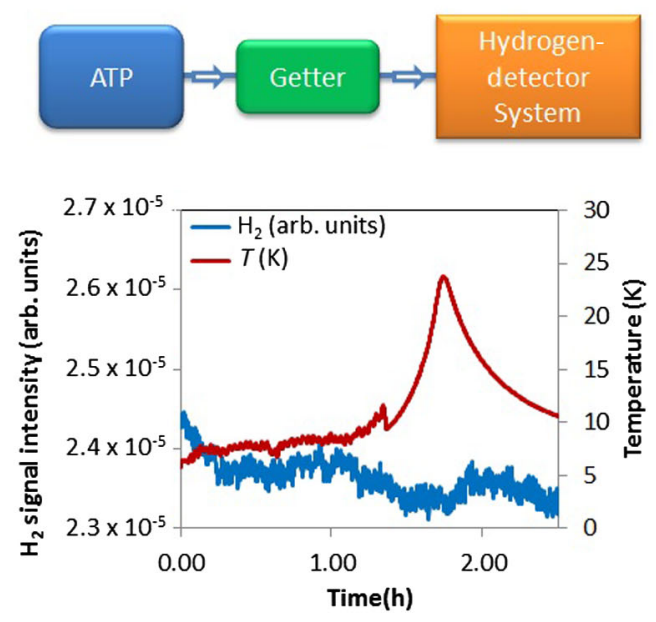

(b)

FIG. 10 On-line monitoring of $\mathrm{H}_{2}$ content in the $\mathrm{He}(\mathrm{g})$ at the outlet of ATP (a) and getter material (b).

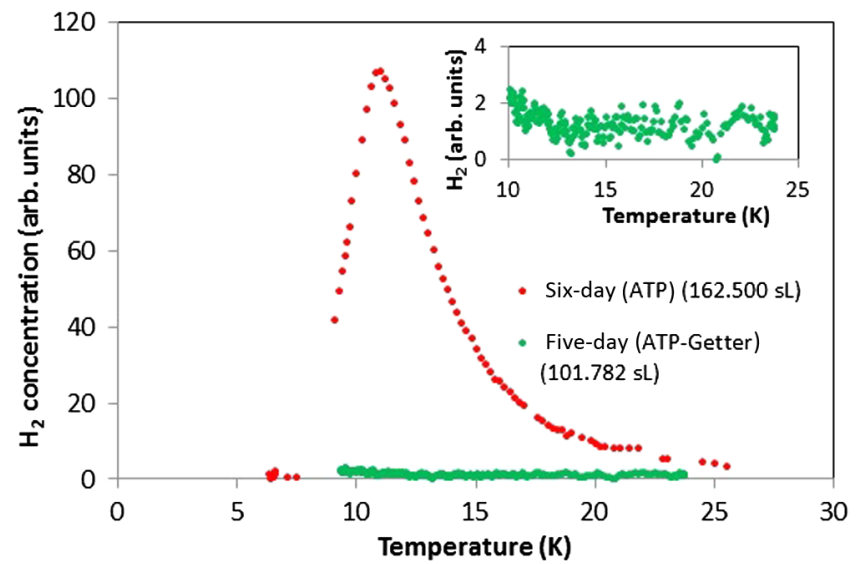

FIG. 11. Red circles: $\mathrm{H}_{2}$ concentration in arbitrary units after about six days purifying recovered helium in a SS HRP recovery plant without a getter element. Green circles: $\mathrm{H}_{2}$ concentration after five days purifying recovered green helium gas. The amount of purified gas is of the order of $10^{5} \mathrm{sL}$ in both cases.

The liquid produced with green helium gas does not contain floating solid hydrogen; thus, the surface is optically clean, as can be seen in Video 2.

\section{SOLUTIONS FOR LARGE-SCALE HELIUM RECOVERY PLANTS: HIGH-PRESSURE GREEN HELIUM LIQUEFACTION PLANTS}

Large installations are prone to $\mathrm{H}_{2}$ accumulation up to problematic levels over time [34]. Typically, the metallic piping of recovery systems and the storage cylinders outgas $\mathrm{H}_{2}$. Also, oils in pumps and compressors will produce $\mathrm{H}_{2}$ when heated. If the central liquid storage is kept cold and full for a long time without routinely warming all content, $\mathrm{H}_{2}$ will accumulate inside it until it reaches critical levels.

In Leiden, we install a catalytic unit with the purpose of removing $\mathrm{H}_{2}$ traces from the recovered helium just before it enters the liquefaction unit(s). This is a DeOxo commercially available (BASF) catalyst specifically designed for the removal of $\mathrm{O}_{2}$ and/or $\mathrm{H}_{2}$ from gas streams at room

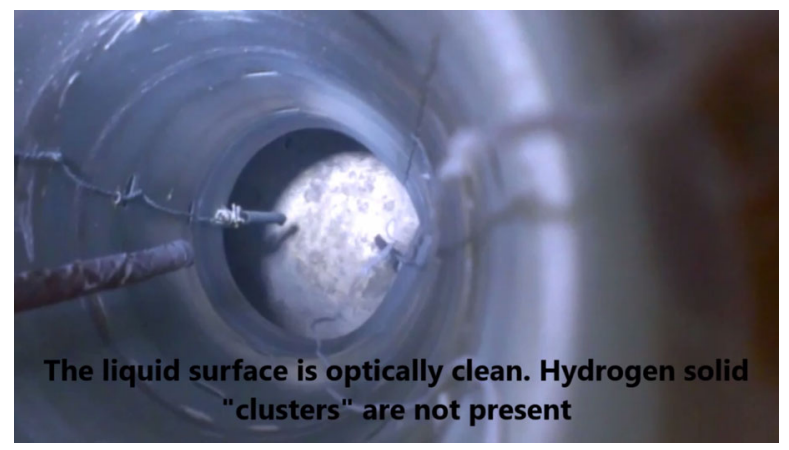

VIDEO 2. Top view of a liquid-helium Dewar through a methacrylate cover. The video shows liquid produced with green helium gas. 


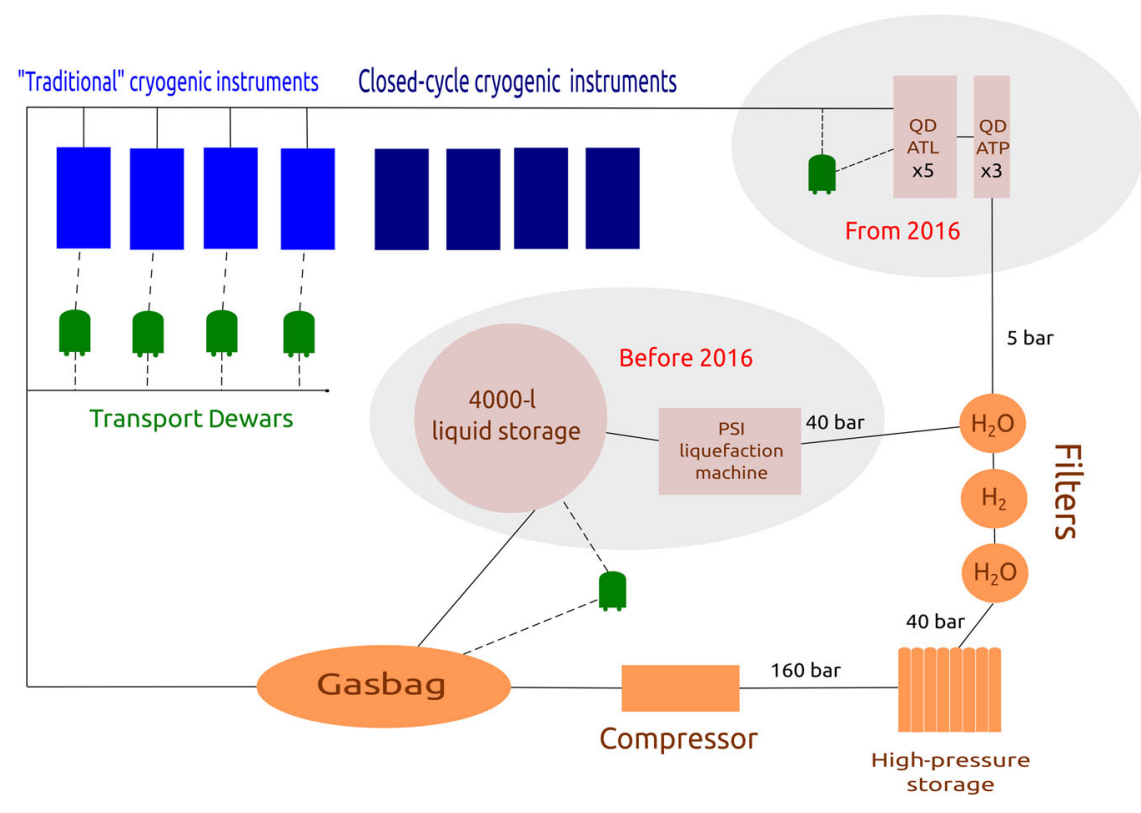

FIG. 12. Leiden Institute of Physics cryogenic installation. Before 2016, there was one single liquefaction machine, a PSI $\mathrm{KOCH}$ liquefier, connected to a large storage $(40001)$ of liquid helium. Helium gas from the recovery system is purified from oils, water, and hydrogen traces, and it enters the liquefaction unit as helium $99.8 \%$, where the residual contamination is air. Air (nitrogen and oxygen) is removed by cold traps within the liquefier. From 2016, the department has substituted the old machine with five Quantum Design ATL160 small-scale helium liquefiers and three ATP30 purifiers. Helium purified from oils, water, and hydrogen traces, by the same sequence of filters, enters the Quantum Design ATPs, which remove air by cryocondensation before entering the ATLs.

temperature. The hydrogen molecules are dissociative chemisorbed on the catalyst surface and further oxidized to $\mathrm{H}_{2} \mathrm{O}(\mathrm{g})$ by the $\mathrm{O}_{2}$ impurities also present in the incoming gas.

Water produced in the helium stream after the DeOxo is trapped by a $\mathrm{H}_{2} \mathrm{O}$ trap, while excess water present in the helium gas before the DeOxo is filtered by another $\mathrm{H}_{2} \mathrm{O}$ trap (see Fig. 12). DeOxo catalysts can be made with either platinum- or palladium-based precious metals and are typically impregnated on alumina oxide $\left(\mathrm{Al}_{2} \mathrm{O}_{3}\right)$ spheres or pellets. The catalyst can operate from atmospheric pressures up to several hundred pounds per square inch. In Leiden, it is operated at 40 bar. Since the $\mathrm{H}_{2}$ trace filtering system was installed in Leiden in 2010, $\mathrm{H}_{2}$ blocking has not occurred.

\section{CONCLUSIONS}

In conclusion, we propose a plausible mechanism to explain the blockage of capillary tubes used as flow impedances to achieve temperatures below $4.2 \mathrm{~K}$ in ${ }^{4} \mathrm{He}$ evaporation cryostats that are used in many lowtemperature research laboratories. Our experimental work demonstrates that $\mathrm{H}_{2}$ impurities at molar fraction levels in He of the order of $10^{-10}$ are at the origin of this worldwide experienced problem, which has considerable opex costs. Estimates of the blockage time are consistent with the $\mathrm{H}_{2}$ concentrations calculated from the vapor pressure of solid hydrogen at $4.2 \mathrm{~K}$. We develop and implement solutions for both small- and large-scale helium recovery plants, which are proven to be highly effective over periods of years.

\section{ACKNOWLEDGMENTS}

The authors are greatly appreciative and acknowledge the financial support from the Spanish Ministry of
Economy and Competitiveness through the INNPACTO Projects No. IPT-2012-0442-420000 and No. MAT201564083-R, in addition to European Union FEDER funds. They also acknowledge the use of Servicio General de Apoyo a la Investigación-SAI, Universidad de Zaragoza as well as Mr. F. Gómez, Mr. A. Castaño, and Mr. D. Finol for their technical support.

[1] S. C. Collins, Helium refrigerator and liquefier, Adv. Cryog. Eng. 11, 11 (1966).

[2] ATL160 and ATP30 manufactured by Quantum Design, Inc., San Diego, 2016, http://www.qdusa.com/products/ helium-liquefiers.html.

[3] C. Chialvo, T. Sayles, J. Diederichs, S. Spagna, M. Gabal, J. Sesé, and C. Rillo, Smart energy compressors for helium liquefiers, in Proceedings of Cryocoolers 18, edited by S. D. Miller and R. G. Ross, Jr. (International Cryocooler Conference, Inc., Boulder, 2014), http://cryocooler.org/ proceedings/ICC-18-proceedings/Cryocoolers18.htm.

[4] C. Rillo, M. Gabal, M. P. Lozano, J. Sesé, S. Spagna, J. Diederichs, R. Sager, C. Chialvo, J. Terry et al., Enhancement of the Liquefaction Rate in Small-Scale Helium Liquefiers Working Near and Above the Critical Point, Phys. Rev. Applied 3, 051001 (2015).

[5] C. Rillo and L. Tocado, Helium-recovery plant, U.S. Patent No. 8,973,397 (2015), https://patentimages.storage .googleapis.com/pdfs/825e5bae782e3f607ff1/US8973397 .pdf.

[6] C. Wang, Small scale helium liquefaction systems, J. Phys. Conf. Ser. 150, 012053 (2009).

[7] Series $4 \times 10$ QUANTUMCOOLER manufactured by Quantum Technology Corp., Squamish, Canada, 2016, http:// quantum-technology.com/liquify/helium-liquefiers.html.

[8] I. I. Abrikosova and A. I. Shal'nikov, The purity of liquid helium, Cryogenics 11, 137 (1971). 
[9] J. W. Riehl, J. L. Kinsey, J. S. Waugh, and J. H. Rugheimer, Spin-lattice relaxation and the anisotropic part of the $\mathrm{He}-\mathrm{H}_{2}$ intermolecular potential, J. Chem. Phys. 49, 5276 (1968).

[10] J. W. Riehl, C. J. Fisher, J. D. Baloga, and J. L. Kinsey, Spin-lattice relaxation and the anisotropic part of the $\mathrm{H}_{2}-\mathrm{He}$ and $\mathrm{H}_{2}-\mathrm{Ne}$ intermolecular potential, J. Chem. Phys. 58, 4571 (1973).

[11] M. D. Gordon and D. Secrest, Helium-atom-hydrogenmolecule potential surface employing the LCAO-MOSCF and CI methods, J. Chem. Phys. 52, 120 (1970).

[12] I. F. Silvera, Ultimate fate of a gas of atomic hydrogen in a liquid-helium chamber: Recombination and burial, Phys. Rev. B 29, 3899 (1984).

[13] D. F. McFarland, Composition of gas from a well at Dexter, Kan., Trans. Kans. Acad. Sci. 19, 60 (1903).

[14] P. Froehlich and J. J. Clausen, Large scale helium liquefaction and considerations for site services for a plant located in Algeria, AIP Conf. Proc. 985, 549 (2008).

[15] U. Lindemann, S. Boeck, L. Blum, and K. Kurtcuoglu, Turnkey helium purification and liquefaction plant for Darwin, Australia, AIP Conf. Proc. 1218, 271 (2010).

[16] The Future of Helium as a Natural Resource, edited by W. J. Nuttall, R. H. Clarke, and B. A. Glowacki (Routledge, Abingdon, 2012).

[17] Linde Helium Catalog, Linde Gases Division, USA, 2016, http://hiq.linde-gas.com/en/specialty_gases/pure_gas_ finder.html.

[18] Air Liquide Specialty Gases Catalog, Air Liquide Group, France, 2016, https://industry.airliquide.us/helium.

[19] Praxair Helium Catalog, Praxair Technology, Inc., Danbury, 2016, http://www.praxair.com/gases/buy-helium-gas-or-liquidhelium.

[20] P. C. Novelli, P. M. Lang, K. A. Masarie, D. F. Hurst, R. Myers, and J. W. Elkins, Molecular hydrogen in the troposphere: Global distribution and budget, J. Geophys. Res. 104, 30427 (1999).

[21] M. Gabal, M. P. Lozano, A. Oca, M. P. Pina, J. Sesé, and C. Rillo, Helium contamination through polymeric walls, Phys. Procedia 67, 141 (2015).

[22] C. Jewell and P. V. E. McClintock, A note on the purity of liquid helium-4, Cryogenics 19, 682 (1979).
[23] L. E. DeLong, O. G. Symko, and J. C. Wheatley, Continuously operating ${ }^{4} \mathrm{He}$ evaporation refrigerator, Rev. Sci. Instrum. 42, 147 (1971).

[24] N. Dittmar, D. Welker, C. Haberstroh, U. Hesse, and M. Krzyzowski, Operating parameters of liquid helium transfer lines used with continuous flow cryostats at low sample temperatures, IOP Conf. Ser. 101, 012097 (2015).

[25] J. Garside and T. J. Foster, Reduction of blockages in a cryogenic refrigeration system such as for magnetic resonance imaging systems, U.S. Patent No. US 2015/0153427 A1 (2015), https://patentimages.storage.googleapis.com/ pdfs/b378f86d03ca380c4395/US20150153427A1.pdf.

[26] B. N. Engel, G. G. Ihas, E. D. Adams, and C. Fombarlet, Insert for rapidly producing temperatures between 300 and $1 \mathrm{~K}$ in a helium storage Dewar, Rev. Sci. Instrum. 55, 1489 (1984).

[27] A. Züttel, Hydrogen storage methods, Naturwissenschaften 91, 157 (2004).

[28] P. Bénard and R. Chahine, Storage of hydrogen by physisorption on carbon and nanostructured materials, Scr. Mater. 56, 803 (2007).

[29] P. P. P. M. Lerou, H. J. M. ter Brake, H. J. Holland, J. F. Burger, and H. Rogalla, Insight into clogging of micromachined cryogenic coolers, Appl. Phys. Lett. 90, 064102 (2007).

[30] H. S. Cao, S. Vanapalli, H. J. Holland, C. H. Vermeer, and H. J. M. ter Brake, Clogging in micromachined JouleThomson coolers: Mechanism and preventive measures, Appl. Phys. Lett. 103, 034107 (2013).

[31] PPMS manufactured by Quantum Design, Inc., San Diego, 2016, http://www.qdusa.com/products/ppms.html.

[32] R. F. Barron, Cryogenic Systems (Oxford University Press, New York, 1985).

[33] E. W. Lemmon, M. L. Huber, and M. O. McLinden, NIST Standard Reference Database 23: Reference Fluid Thermodynamic and Transport Properties, version 9.1, National Institute of Standards and Technology, 2013, http://www .nist.gov/srd/nist23.cfm.

[34] H. Ikeda and Y. Kondo, Improvement of the operational settings of a helium purifier, leading to a higher purity of the recovered gas, Phys. Procedia 67, 1153 (2015). 